Original article

\title{
Effect of modified tray design on accuracy of different impression techniques for parallel and divergent implants
}

\author{
Liem Dang, Matthew Woliansky, Joseph Palamara, and Jaafar Abduo \\ Restorative Section, Melbourne Dental School, Melbourne University, Melbourne, Australia
}

(Received November 17, 2019; Accepted April 3, 2020)

\begin{abstract}
This study evaluated the effect of a modified tray design on the accuracy of implant impressions in comparison with the non-splinted and splinted impression techniques. Two titanium frameworks were produced to fit two parallel implants and two divergent implants with a $15^{\circ}$ angle. According to the frameworks employed, two acrylic resin master models were fabricated. For each model, 10 impressions were taken with every technique. The maximum framework principal strain was calculated for every generated cast. For the parallel implant model, the strains of the nonsplinted $(118.4 \mu \varepsilon)$, splinted $(89.0 \mu \varepsilon)$, and modified tray design impression $(49.4 \mu \varepsilon)$ techniques were statistically similar $(P=0.16)$. For the divergent implant model, all the impression techniques showed a considerably higher strain than the parallel implant model. The splinted $(287.0 \mu \varepsilon)$ and the modified $(262.9 \mu \varepsilon)$ tray design impression techniques showed similar strains for the divergent implant model, which were significantly less than the strains for the non-splinted impression $(518.0 \mu \varepsilon)$ technique $(P<0.05)$. Therefore, for two parallel implants, all the impression techniques exhibited similar accuracy. When angulation existed between the implants, the splinted and the modified tray design impression techniques were more accurate than the non-splinted impression technique.
\end{abstract}

Keywords; cast, framework, implant impression, impression tray, strain

\section{Introduction}

Implant impression is an integral step for fabrication of any implant prosthesis. An accurate implant impression is mandatory to ensure the prosthesis is precisely seated on the implant. Inaccuracy in the fitting of implant prostheses has been related to numerous biological and mechanical complications such as plaque accumulation, inflammation, peri-implant bone loss, screw loosening, chipping of the veneering material and component wear [1,2]. Previous studies have shown that the accuracy of an impression is influenced by a variety of factors such as the impression concept, impression material, components, implant depth and orientation, the number of implants and prosthesis span [3-6]

To improve the accuracy of multiple implant impressions, several techniques have been proposed, such as abrading of the impression copings, application of tray adhesive to the impression copings, and splinting of the impression copings [3-6]. Splinting with rigid material has received considerable attention as it connects the impression copings and maintains their spatial position within the impression, and subsequently during implant replica fitting and cast pouring. Several laboratory studies have shown that splinted impressions have a general tendency to be more accurate than non-splinted impressions $[3,5,6]$. However, intraoral splinting of impression copings is time consuming, and thus inconvenient for both the patient and the clinician. Furthermore, this procedure is challenging for posterior implants due to limitations of accessibility, and several reports have indicated that splinting is still susceptible to error due to contraction of the self-cured resin material $[7,8]$.

In situations where two parallel implants are required, a recent study

Correspondence to Dr. Jaafar Abduo, Restorative Section, Melbourne Dental School, Melbourne University, 720 Swanston Street, Melbourne, Victoria 3010, Australia

Fax:+61-3-9341-1599 E-mail: jaafar.abduo@unimelb.edu.au

J-STAGE Advance Publication: September 9, 2020

Color figures can be viewed in the online issue at J-STAGE.

doi.org/10.2334/josnusd.19-0482

DN/JST.JSTAGE/josnusd/19-0482 has reported the superior accuracy of a modified tray design where the custom tray is altered to increase the support of the impression material around copings [8]. This modification involves the addition of a "chimney" around each impression coping to ensure that the rigid tray material surrounds the external surfaces of the coping. This design also ensures that a uniform amount of impression material surrounds the copings. Clinically, the new tray design has multiple potential advantages such as ease of use, simplicity, time efficiency, convenience and less material usage in comparison to the splinting technique. Clinically, however, it is frequently observed that the implants are not parallel and have an angle between them that imposes an extra challenge on the impression, as illustrated in multiple studies [5,9-12]. Thus, before a modified tray design can be recommended as a replacement for splinting, it is important to evaluate its accuracy in scenarios that are clinically more relevant, such as the presence of a moderate angle between the implants. The present study was therefore designed to investigate the effect of a modified tray design on the accuracy of implant impressions in comparison with non-splinted and splinted impression techniques for two parallel and divergent implant situations. The null hypothesis was that the difference in impression techniques would not influence the accuracy of parallel and divergent implant impressions.

\section{Materials and Methods}

\section{Master frameworks and model fabrication}

Two master frameworks of grade 5 titanium alloy (Copra Ti-5, Whitepeaks Dental Solutions GmbH \& Co. KG, Essen, Germany) were produced via computer-aided design and computer-aided milling (CAD/CAM) by a commercial manufacturer (Osteon Medical, Mulgrave, Australia). One of the frameworks was designed to fit on two parallel implants, and the other to fit on two divergent implants, one of which was tilted $15^{\circ}$ in the buccolingual direction. The two frameworks had a similar bar-shape design with a cross-sectional height and width of $3 \mathrm{~mm}$, and virtually designed to fit a Straumann regular implant with a neck diameter of $4.8 \mathrm{~mm}$ (Institut Straumann AG, Basel, Switzerland). The implants were $15 \mathrm{~mm}$ apart from their centers.

Two master models were designed to have an alveolar ridge shape and indexed with notches to serve as distinct and reproducible stops during seating of the tray. The ridge width was $8 \mathrm{~mm}$ at the crest and $14 \mathrm{~mm}$ at the base (Fig. 1). The master models were fabricated from self-curing polymethylmethacrylate resin (Vertex Selfcuring Resin; Henry Schein, Waterloo, Australia). Two implants were attached on each framework, and subsequently inserted into unset self-curing polymethylmethacrylate resin within drilled holes on the crests of the master models. This ensured the most accurate fit of the frameworks on the implants within each master model. Subsequently, the frameworks were utilized to measure the accuracy of each produced cast $[13,14]$. The platforms of the implants were placed $1 \mathrm{~mm}$ above the crest, and the undercuts below the implant shoulders were completely sealed with the acrylic resin material. This prevented excessive distortion of the impression material and facilitated connection of the implant replicas on the impression copings.

\section{Tray fabrication}

For every tray design, two layers of baseplate wax $1.5 \mathrm{~mm}$ thick (Truewax, Dentsply, Ballantyne Corporate $\mathrm{Pl}$, Charlotte, NC, USA) were applied on each master model to form a consistent spacer between the master model and the tray. The trays were fabricated from light-cured acrylic resin material (Vertex Dental, Soesterberg, Netherlands). Every effort was made to 

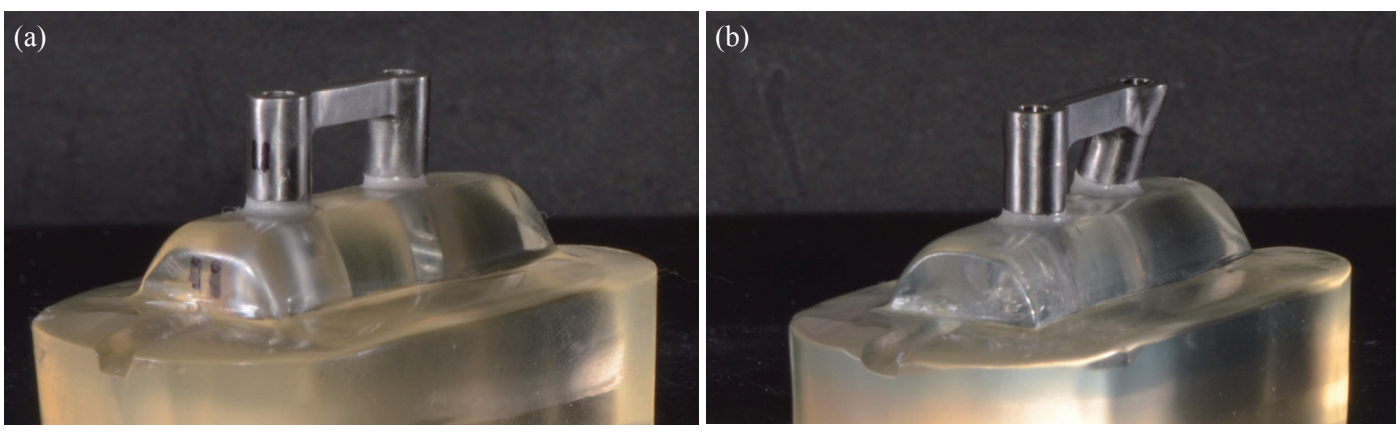

Fig. 1 The master frameworks and models used in the study. (a) Parallel implants. (b) Divergent implants

(a)

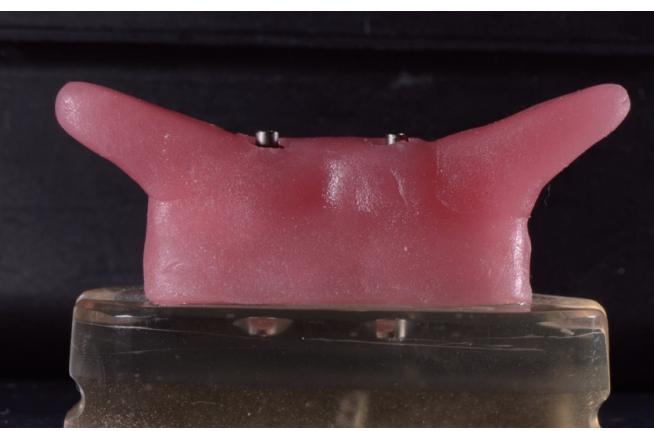

(b)

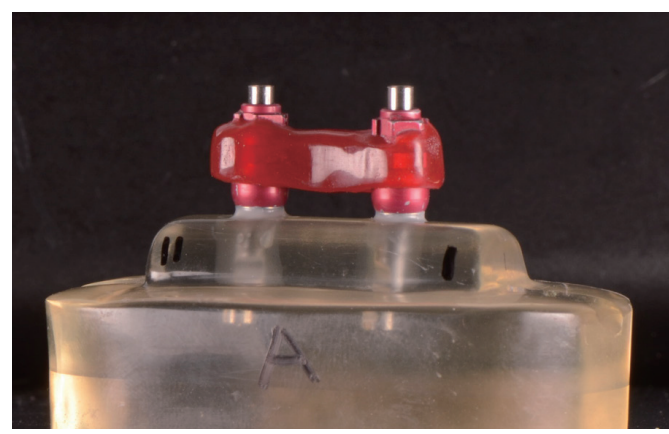

(c)

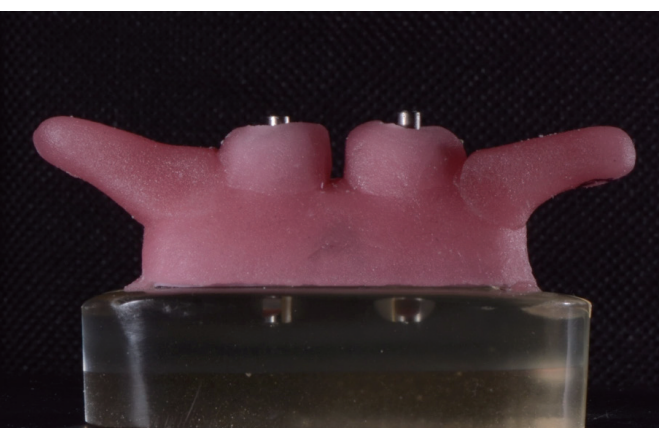

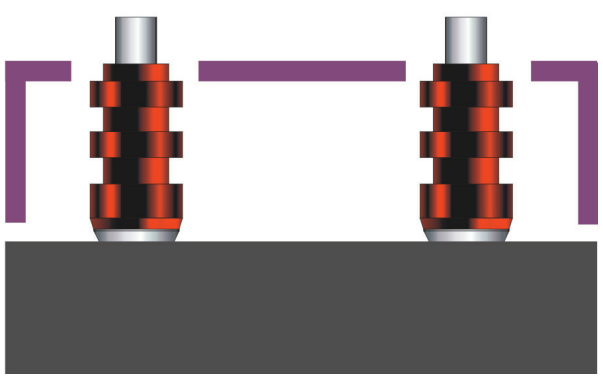
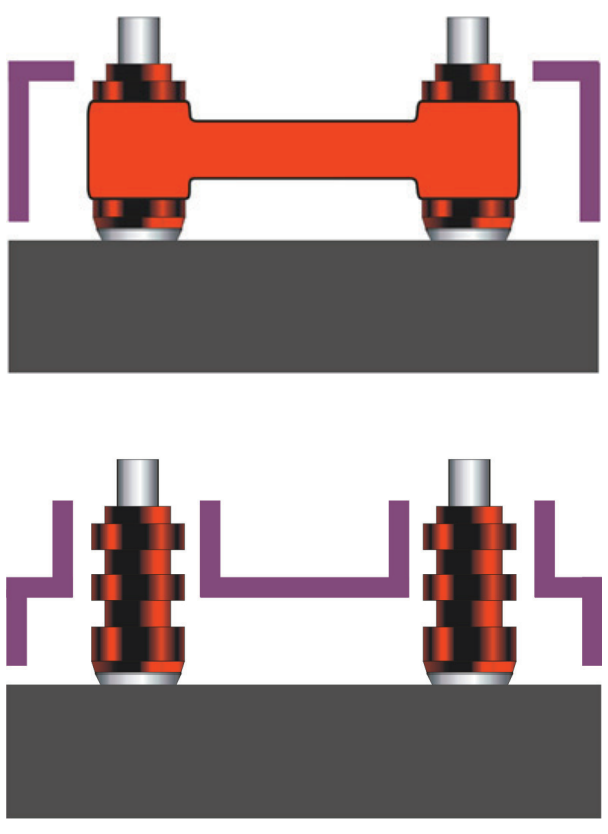

Fig. 2 Images of the different impression techniques. (a) The non-splinting impression technique using an open custom tray. (b) The splinting impression technique with the resin splint connecting the impression copings. (c) The modified tray impression technique with the custom tray material forming chimneys that surround most of the impression copings

ensure all the trays for all the techniques had similar dimensions except around the implant sites. Two handles were attached to each tray on the mesial and distal sides to help with handling, and all the trays were indexed at their bases to fit against the master models.

For the non-splinting tray design, two circular openings that corresponded with the implant locations were made in the occlusal aspect of the trays (Fig. 2a). A space of approximately $2 \mathrm{~mm}$ was allowed between the external surface of the copings and the internal surface of the tray. For the splinting tray design, the two circular openings were joined to accommodate the splint between the impression copings (Fig. 2b). The modified tray design was distinguished with two vertical openings resembling chimneys to entirely cover the impression copings (Fig. 2c). The space between the chimney of the tray and the impression copings was approximately 1.5-2.0 $\mathrm{mm}$, which corresponded to the thickness of a single baseplate wax sheet. To ensure the trays were fabricated with similar dimensions, the waxed master model was duplicated using laboratory putty. The putty duplicate was then used to fabricate all the trays. A total of 10 trays were produced for every impression technique and master model.

\section{Impressions}

For every impression technique for each master model, 10 implant level impressions were obtained. The intaglio surfaces of the trays, and the impression copings were coated with tray adhesive (VPS Tray adhesive, Kerr Corporation, Orange, CA, USA), and baseplate wax was placed over the tray openings. The splinted impression technique involved splinting the two impression copings with self-curing acrylic resin material (GC Pattern Resin, GC Corp., Tokyo, Japan). The resin splints were designed to be at least $2 \mathrm{~mm}$ thick around the impression copings and with a minimal body thickness of $3 \mathrm{~mm}$. To reduce the possible effects of polymerization shrinkage, the splints were left for 24 hours, sectioned, and reconnected 
Table 1 Summary of the generated strain values for the parallel implant model and the divergent implant model

\begin{tabular}{|c|c|c|c|c|c|c|}
\hline & \multicolumn{3}{|c|}{ Parallel implant model } & \multicolumn{3}{|c|}{ Divergent implant model } \\
\hline & Non-splinted & Splinted & Modified tray & Non-splinted & Splinted & Modified tray \\
\hline Median $(\mu \varepsilon)$ & 118.4 & 89.0 & 49.4 & 518.0 & 287.0 & 262.9 \\
\hline Interquartile range $(\mu \varepsilon)$ & 90.9 & 34.4 & 86.1 & 159.2 & 226.7 & 325.8 \\
\hline Mean $(\mu \varepsilon)$ & 170.4 & 98.2 & 98.7 & 535.0 & 309.1 & 288.0 \\
\hline $\mathrm{SD}(\mu \varepsilon)$ & 145.4 & 43.8 & 101.5 & 155.6 & 226.2 & 215.2 \\
\hline Maximum $(\mu \varepsilon)$ & 528.6 & 193.1 & 351.0 & 755.0 & 777.1 & 584.2 \\
\hline Minimum $(\mu \varepsilon)$ & 61.3 & 34.3 & 20.1 & 239.9 & 27.3 & 30.4 \\
\hline
\end{tabular}

(a)

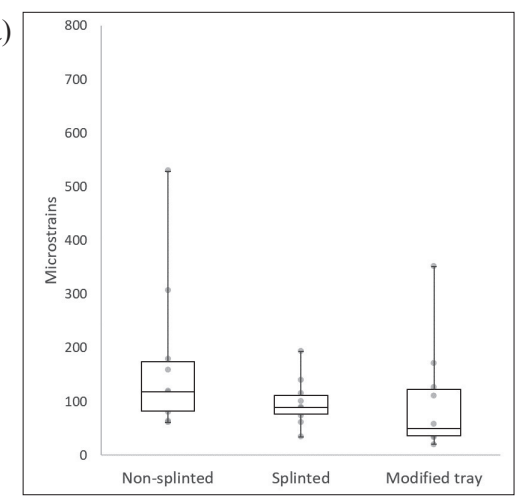

(b)

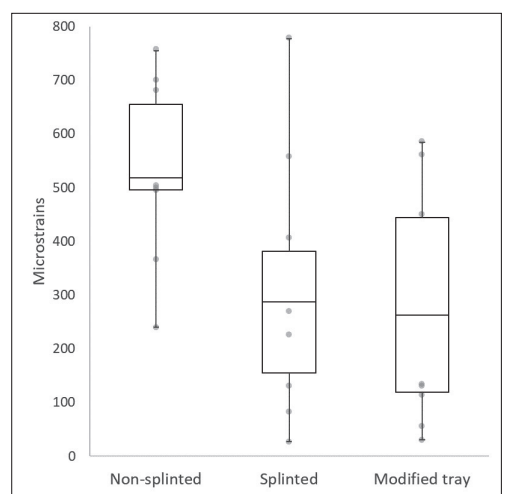

Fig. 3 Box-plot diagrams of the maximum principal strain distributions. (a) Parallel implants model. (b) Divergent implants model

by a newly mixed resin $[5,8,14,15]$. All impressions were taken using heavy body polyvinylsiloxane (PVS) impression material (Kerr Extrude Extra type 1, Kerr Corporation), and excess material was wiped away by hand. Implant replicas were attached to the impression copings and type IV dental stone (GC Fujirock EP, GC Corp.) was used for pouring the impressions to construct the test casts, which were left for 24 hours prior to removal.

\section{Strain analysis}

Strain analysis was conducted to evaluate the accuracy of fit for each master framework on the test casts. Large strain magnitudes indicate a greater level of master framework misfit, which can be attributed to implant impression distortion. A 3-stacked foil rosette strain gauge (Vishay Precision Group, Raleigh, NC, USA) was attached to the middle of each titanium framework on the occlusal surface using cyanoacrylate resin $(\mathrm{M}$ Bond 200 adhesive, Vishay Micro-measurements, Raleigh, NC, USA). The framework occlusal surface was chosen because it is parallel to the testing bench regardless of the implant location, which allowed similar placement of the strain gauges on the two frameworks. The strain gauge foils had a length of $1.0 \mathrm{~mm}$, a width of $0.7 \mathrm{~mm}$, a resistance of $120 \pm$ $0.5 \% \Omega$, and a gauge factor of 2.1. The strain gauges were protected with a silicone coat (RTV Coating, Dow Corning Corp, Midland, MI, USA) to prevent displacement of the foils after each test. Prior to manual fitting of the framework on each sample cast, the baseline microstrain $(\mu \varepsilon)$ reading was set to zero to standardize the measurements. On each test cast, one framework screw was hand-tightened until initial resistance was felt, followed by similar hand tightening of the other screw. This was followed by torqueing the retaining screws to $35 \mathrm{Ncm}$ according to the same tightening sequence. The readings following screw torqueing of each foil were recorded in $\mu \varepsilon$ by a Wheatstone bridge electrical circuit data acquisition system (Acquire N Scans, National Instruments Corp., Austin, TX, USA). Through the testing, strain developments were observed using computer software (Lab View 7 Express, National Instruments Corp.). For each test cast, the strain evaluations were repeated with a reversed screw tightening sequence. Since the rosette strain gauge is composed of 3 foils $\left(0^{\circ}, 45^{\circ}\right.$ and $90^{\circ}$ orientations), the acquisition system recorded the magnitude of strain from each foil separately. The strain values from the 3 foils were imported to Mohr's circle equation to calculate the maximum principal strain magnitude:

Maximum principal strain $=\frac{\varepsilon_{1}+\varepsilon_{3}}{2}+\frac{1}{\sqrt{2}} \sqrt{\left(\varepsilon_{1}-\varepsilon_{2}\right)^{2}+\left(\varepsilon_{2}-\varepsilon_{3}\right)^{2}}$

where $\varepsilon_{1}$ is the $0^{\circ}$ foil strain, $\varepsilon_{2}$ is the $90^{\circ}$ foil strain, and $\varepsilon_{3}$ is the $45^{\circ}$ foil strain.

Since two strain readings were obtained with the reversed screw tight- ening sequence for each test cast, two maximum principal strain values were generated. Eventually, the two values were averaged to generate a single maximum principal strain value for each test cast.

\section{Statistics}

The median and interquartile range of the maximum principal strain values for each technique of every model were calculated. The Shapiro-Wilks test was used to assess the normality of the outcome variables. The KruskalWallis test was applied for each master model to evaluate the significance of differences among the different impression techniques followed by the Bonferroni correction test for pairwise comparisons. In addition, the effect of parallel and divergent implant models on the accuracy of each impression technique was evaluated by the Mann-Whitney $U$-test. All statistical analyses were conducted using a statistics program (SPSS for Windows, v23; SPSS Inc, Chicago, IL, USA). The level of significance was set at 0.05 .

\section{Results}

The data obtained using all the above techniques are summarized in Table 1. The Shapiro-Wilks test indicated that some data were not normally distributed $(P<0.05)$, which confirmed the suitability of the KruskalWallis test. The median (interquartile range) maximum principal strain values for parallel implants were $118.4 \mu \varepsilon(90.9 \mu \varepsilon), 89.0 \mu \varepsilon(34.4 \mu \varepsilon)$ and $49.4 \mu \varepsilon(86.1 \mu \varepsilon)$ for the non-splinted, splinted, and modified tray impression techniques, respectively. There were no significant differences among the techniques when the implants were parallel $(P=0.16)$. For divergent implants, the greatest maximum principal strains were associated with the non-splinted technique $(518.0 \mu \varepsilon$ [159.2 $\mu \varepsilon])$, followed by the splinted $(287.0 \mu \varepsilon[226.7 \mu \varepsilon])$ and the modified tray $(262.9 \mu \varepsilon[325.8$ $\mu \varepsilon])$ techniques. The differences among the techniques were significant for the divergent implant model $(P=0.03)$. However, significant differences were evident between the non-splinted and the splinted techniques $(P=$ $0.04)$, and the non-splinted and the modified tray techniques $(P=0.03)$. No significant difference was observed between the splinted and the modified tray techniques $(P=0.96)$.

The divergent implant model was associated with significantly more maximum principal strain than the parallel implant model for the nonsplinted $(P=0.00)$, the splinted $(P=0.02)$, and the modified tray $(P=$ $0.03)$ techniques. Regardless of the impression technique employed, an almost 3-fold increase in maximum principal strain was observed for the divergent than for the parallel implant model. The distributions of the maximum principal strain values for the parallel and divergent implants are summarized in box plot diagrams (Fig. 3). In general, for the parallel 
implant model, the non-splinted technique showed more variation than the other techniques. The variations in the maximum principal strain values for the divergent implant model were similar among all the techniques, although the non-splinted technique showed more skewing towards larger values.

\section{Discussion}

The present findings indicate that the impression technique has minimal influence on the accuracy of the impression when the two implants are parallel. Thus, the hypothesis that the accuracies of all the impression techniques would be similar was accepted for the parallel implant model. However, a buccolingual divergence of $15^{\circ}$ led to a more prominent effect of the different impression techniques, the modified tray and the splinted techniques being equally superior to the non-splinted technique. As a result, the hypothesis that the different techniques would have similar accuracy was rejected for the divergent implant model, suggesting that when impressions of multiple diverging implants are taken, a modified impression technique should be adopted to ensure accurate prosthesis fabrication.

For parallel implants, all the techniques were associated with discrepancies of a similar magnitude that were attributable to accumulation of errors from all the steps and the materials employed [6]. For example, for the non-splinted and modified tray techniques, errors can occur during material setting, impression removal, displacement of the copings within the set impression material, fitting of implant replicas and setting of the dental stone [16]. Likewise, even for the splinted technique, the splint resin material employed can introduce errors due to polymerization shrinkage of the resin [7]. However, as additional steps, such as sectioning and reattachment, were employed to reduce these errors $[6,7,14,15]$, it can be speculated that all of the techniques are of equally acceptable accuracy for two parallel implants. Although the effects of splinting on impression accuracy have been intensively investigated, the outcomes have been inconsistent: some studies found that splinting was superior [6,15-17] whereas others did not confirm this $[4,13,14]$. This variability in the outcome may have been due to variations in the splinting protocol, splinting material, splint rigidity, implant connection, and distance between implants $[7,18]$. However, in accordance with the present results, several earlier laboratory studies reported that the impression technique had a minimal effect on the accuracy of two-implants impressions when they were parallel $[13,14]$. Specifically, a frequent observation was that the accuracy of the splinting technique was similar to that of the non-splinting technique $[8,14]$. The similarity of outcomes among the techniques may be due to the fact that the parallel implant model is the least challenging of any implant impression technique [12]. The vertical path of removal minimizes strains within the set elastomeric impression material during removal of the impression from the model, and as a result the final impression is less likely to suffer permanent deformation. As soon as the impression technique is challenged in a way that will distort the material, accuracy differences between the techniques may become obvious. These challenges can be due to the presence of teeth adjacent to implants, an increased number of implants, or alterations to implant orientation [4-6,17]. Therefore, in accordance with earlier observations, it can be speculated when the implants are parallel, one specific technique has no advantage over another [10-12].

When angulation was introduced between implants, all the impression techniques showed greater errors than for parallel implants. However, the non-splinted technique showed the greatest deterioration of accuracy. Several authors have confirmed that the presence of an angle between implants is associated with greater impression error [5,9-12]. For example, Chia et al. found a consistent increase in error if the angle between implants was increased to $10^{\circ}$ and to $20^{\circ}$ [10]. Likewise, Parameshwari et al. reported increased errors when the implant angulation was increased to $15^{\circ}$ and to $25^{\circ}[12]$. This is attributed to differences in the paths of withdrawal, which would make impression removal more difficult and subject the impression material to more strain as the impression copings disengage the implants. Eventually, this would lead to inevitable coping displacement within the set impression material [9-12]. Specifically, Assuncao et al. reported that more errors occurred around an angulated implant than around a parallel implant [9]. Although all impression techniques were associated with greater errors for divergent implants than for parallel implants, the splint- ing and the modified tray design was clear advantageous. The benefit of a resin splint is related to rigid fixation of the impression coping, rendering it more capable of maintaining the 3D position of the implant and preventing individual coping displacement during impression-taking, fitting of implant replicas and impression pouring [16]. This is in accordance with several earlier studies of divergent implants, which showed that the splinting technique yielded significantly more accurate impressions than the non-splinted technique $[9,11,19]$.

The superior outcome of the modified tray design can be attributed to the extension and encirclement of the impression copings by the rigid tray material [8]. This coverage ensured that the impression copings and the surrounding impression material were rigidly supported. This may have increased the positional stability of the impression copings through the impression-taking and casting processes. Earlier studies have reported that the more rigid custom trays may yield implant impressions with greater accuracy than stock trays that are less rigid $[12,20,21]$. The positive effect of rigid tray support for elastomeric impression materials was also confirmed by several laboratory studies [22-25]. In addition, the modified tray design is advantageous in reducing the bulk of the impression material surrounding the impression copings [8]. This may reduce the magnitude of the total distortion of the impression material $[22,23]$. Several laboratory studies have shown that most of the elastomeric impression material bulk is associated with greater shrinkage and subsequent inaccuracies in the impressions [26,27]. Eventually, as the modified tray design reduces the bulk of the impression material around the coping, the likelihood of errors in coping orientation is reduced to a similar level to that of the splinting technique. This may have clinical relevance as the modified tray technique is simpler, less time consuming and more convenient for both the clinician and patient than the splinting technique.

Although the present results suggest the superiority of the modified tray and the splinted techniques over the non-splinted technique for divergent implants, the clinical significance of this outcome is yet to be confirmed. While strain gauge analysis is an accurate laboratory approach for measurement of error and distortion, it does not quantify the actual dimensional error. Furthermore, strain analysis has a limitation in being sensitive to strain gauge location, overheating and manual handing of the framework $[8,13,14]$. Previous clinical studies that compared the accuracy of impressions in a clinical setting did not prove the superiority of one technique over the other $[28,29]$. This may have been due to differences among the laboratories and the clinical set-ups employed for taking the impressions. The present laboratory study did not simulate parameters associated with clinical practice such as the presence of teeth, saliva, ridge undercuts and intraoral access, which can influence the handing of implant impressions and subsequently the impression accuracy. These differences limit extrapolation of the present data to the clinical environment. In addition, the outcome is obviously more relevant to two parallel implants and implants with a divergence of up to $15^{\circ}$. Differences in angulation and the number of implants should therefore be validated in additional studies. Furthermore, since the models in the present study used implants with shallow internal connection, the outcome cannot be generalized to implants with deep conical connections [5].

Therefore, while considering the limitations of the present laboratory study, it can be concluded that for parallel implants, all of the impression techniques used yielded similar accuracy. When a divergence of $15^{\circ}$ existed between implants, all the impression techniques demonstrated deterioration of the accuracy. For divergent implants, the modified tray and the splinted techniques were superior to the non-splinted technique, although there was no difference in accuracy between the techniques.

\section{Acknowledgments}

The authors would like to acknowledge the technical support of Mr Attila Gergely in developing the master models and the custom trays. This study was supported by Melbourne Dental School Research Higher Degree Funding.

\section{Conflict of interest}

The authors have no conflict of interest to declare. 


\section{References}

1. Schwarz MS (2000) Mechanical complications of dental implants. Clin Oral Implants Res $11,156-158$.

2. Abduo J, Judge RB (2014) Implications of implant framework misfit: a systematic review of biomechanical sequelae. Int J Oral Maxillofac Implants 29, 608-621.

3. Lee H, So JS, Hochstedler JL, Ercoli C (2008) The accuracy of implant impressions: a systematic review. J Prosthet Dent 100, 285-291

4. Baig MR (2014) Multi-unit implant impression accuracy: a review of the literature. Quintessence Int 45, 39-51.

5. Papaspyridakos P, Chen CJ, Gallucci GO, Doukoudakis A, Weber HP, Chronopoulos V (2014) Accuracy of implant impressions for partially and completely edentulous patients: a systematic review. Int J Oral Maxillofac Implants 29, 836-845.

6. Moreira AH, Rodrigues NF, Pinho AC, Fonseca JC, Vilaca JL (2015) Accuracy comparison of implant impression techniques: a systematic review. Clin Imp Dent Relat Res 17, 751764.

7. Cerqueira NM, Ozcan M, Goncalves M, da Rocha DM, Vasconcellos DK, Bottino MA et al. (2012) A strain gauge analysis of microstrain induced by various splinting methods and acrylic resin types for implant impressions. Int J Oral Maxillofac Implants 27, 341-345.

8. Liu DY, Cader FN, Abduo J, Palamara J (2019) Accuracy of different implant impression techniques: evaluation of new tray design concept. J Prosthodont 28, 682-687.

9. Assuncao WG, Tabata LF, Cardoso A, Rocha EP, Gomes EA (2008) Prosthetic transfer impression accuracy evaluation for osseointegrated implants. Imp Dent 17, 248-256.

0. Chia VA, Esguerra RJ, Teoh KH, Teo JW, Wong KM, Tan KB (2017) In vitro threedimensional accuracy of digital implant impressions: the effect of implant angulation. Int J Oral Maxillofac Implants 32, 313-321.

11. Elshenawy EA, Alam-Eldein AM, Abd Elfatah FA (2018) Cast accuracy obtained from different impression techniques at different implant angulations (in vitro study). Int J Implant Dent 4, 1-9.

12. Parameshwari G, Chittaranjan B, Sudhir N, Anulekha-Avinash CK, Taruna M, Ramureddy M (2018) Evaluation of accuracy of various impression techniques and impression materials in recording multiple implants placed unilaterally in a partially edentulous mandible- an in vitro study. J Clin Exp Dent 10,388-395.

13. Inturregui JA, Aquilino SA, Ryther JS, Lund PS (1993) Evaluation of three impression techniques for osseointegrated oral implants. J Prosthet Dent 69, 503-509.

14. Choi JH, Lim YJ, Yim SH, Kim CW (2007) Evaluation of the accuracy of implant-level impression techniques for internal-connection implant prostheses in parallel and divergent models. Int J Oral Maxillofac Implants 22, 761-768.
15. Cabral LM, Guedes CG (2007) Comparative analysis of 4 impression techniques for implants. Implant Dent 16, 187-194.

16. Kim S, Nicholls JI, Han CH, Lee KW (2006) Displacement of implant components from impressions to definitive casts. Int J Oral Maxillofac Implants 21, 747-755.

17. Tarib NA, Seong TW, Chuen KM, Kun MS, Ahmad M, Kamarudin KH (2012) Evaluation of splinting implant impression techniques: two dimensional analyses. Eur J Prosthodont Restor Dentistry 20, 35-39.

18. Del Acqua MA, Chavez AM, Castanharo SM, Compagnoni MA, Mollo Fde A Jr (2010) The effect of splint material rigidity in implant impression techniques. Int J Oral Maxillofac Implants 25, 1153-1158.

19. Carr AB (1991) Comparison of impression techniques for a five-implant mandibular model. Int J Oral Maxillofac Implants 6, 448-455.

20. Burns J, Palmer R, Howe L, Wilson R (2003) Accuracy of open tray implant impressions: an in vitro comparison of stock versus custom trays. J Prosthet Dent 89, 250-255.

21. Del'acqua MA, de Avila ED, Amaral AL, Pinelli LA, de Assis Mollo F Jr (2012) Comparison of the accuracy of plastic and metal stock trays for implant impressions. Int J Oral Maxillofac Implants 27, 544-550.

22. Gordon GE, Johnson GH, Drennon DG (1990) The effect of tray selection on the accuracy of elastomeric impression materials. J Prosthet Dent 63, 12-15.

23. Rueda LJ, Sy-Munoz JT, Naylor WP, Goodacre CJ, Swartz ML (1996) The effect of using custom or stock trays on the accuracy of gypsum casts. Int J Prosthodont 9, 367-373.

24. Millstein P, Maya A, Segura C (1998) Determining the accuracy of stock and custom tray impression/casts. J Oral Rehabil 25, 645-648.

25. Ceyhan JA, Johnson GH, Lepe X (2003) The effect of tray selection, viscosity of impression material, and sequence of pour on the accuracy of dies made from dual-arch impressions. J Prosthet Dent 90, 143-149.

26. de Araujo PA, Jorgensen KD (1985) Effect of material bulk and undercuts on the accuracy of impression materials. J Prosthet Dent 54, 791-794.

27. Kumar V, Aeran H (2012) Evaluation of effect of tray space on the accuracy of condensation silicone, addition silicone and polyether impression materials: an in vitro study. J Indian Prosthodont Soc 12, 154-160.

28. Gallucci GO, Papaspyridakos P, Ashy LM, Kim GE, Brady NJ, Weber HP (2011) Clinical accuracy outcomes of closed-tray and open-tray implant impression techniques for partially edentulous patients. Int J Prosthodont 24, 469-472.

29. Perez-Davidi M, Levit M, Walter O, Eilat Y, Rosenfeld P (2016) Clinical accuracy outcomes of splinted and nonsplinted implant impression methods in dental residency settings. Quintessence Int 47, 843-852. 